\title{
Right atrial lithomyxoma with extramedullary hematopoiesis
}

A 69 year old man was admitted for syncope during physical activity. The history and physical examination was unremarkable. Echocardiographic studies showed normal ventricular dimensions, conserved systolic function, normal left atrium dimension, and normal valvar structures. A rounded mass of heterogenic density was found in the right atrium. The mass arose in the lateral wall and prolapsed through the tricuspid valve during diastole.

The patient underwent surgery for removal of the mass, and he has since remained asymptomatic

The excised specimen measured $6 \times 4 \mathrm{~cm}$, and weighed $40 \mathrm{~g}$. Grossly it was sessile, solid, oval shaped, and had a smooth surface (panel A). Foci of haemorrhage were present throughout the myxoma. The cut surface was grey-white with yellow coloured areas and a stone-like consistency (panel B).

Histological examination revealed that the mass comprised polygonal myxoma cells embedded in a myxoid matrix of acid mucopolysaccharide. No atypias or mitosis were found. Massive bone metaplasia was evident (panel 1), with extramedullary haematopoiesis (panel 3) resembling the appearance of normal trabeculae (panel 2).

Myxoma is the most frequent primary tumour of the heart, arising from the endocardium as a pedunculated mass. Mostly they are found in the left atrium (82\%).

Lithomyxoma is a rare pathological entity, first described by Strousse in 1938. Although
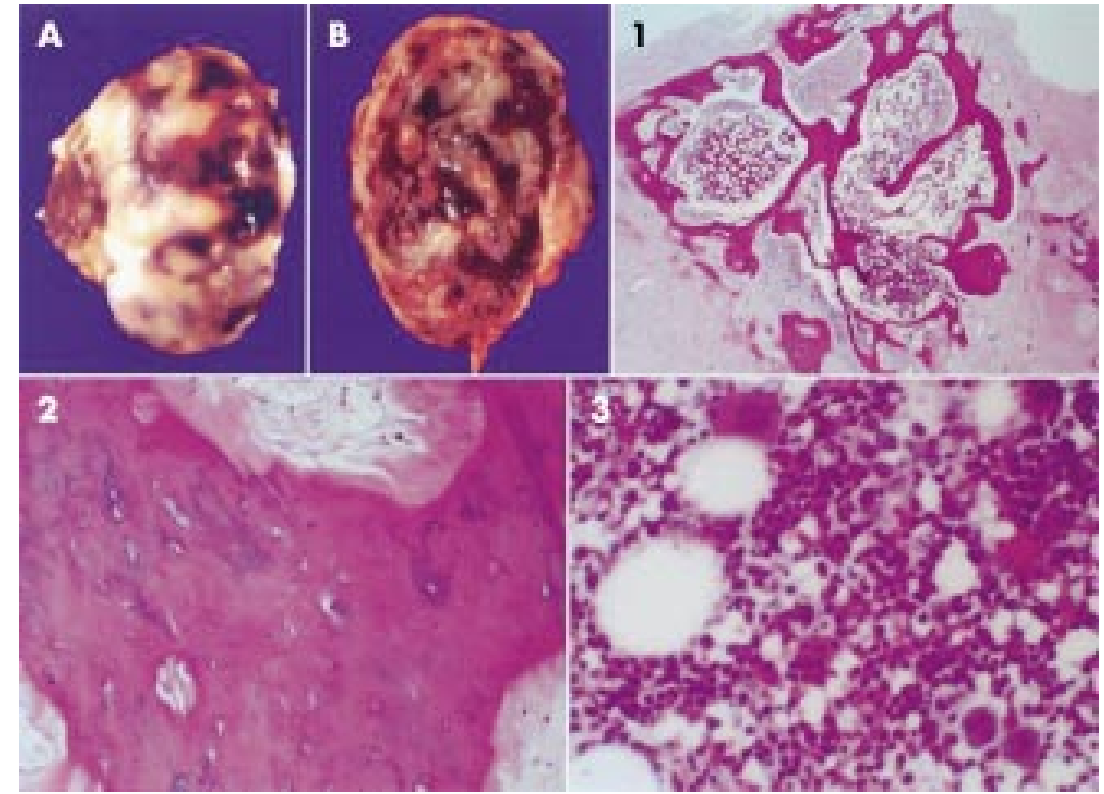

foci of microscopic calcification and areas of metaplastic bone occasionally occur, massive ossification is a rare event; current literature reports 10 cases of lithomyxoma.

Calcification may be considered as a degenerative, age related phenomenon, caused by haemodynamic factors.
Nevertheless, the pathogenesis of cardiac lithomyxoma remains unknown, and further studies are required.

J Azúa-Romeo E Moreno J P Gomollón javierazua@hotmail.com

\section{Diagnosis of a rare cause of arrhythmogenic syncope by means of an implantable loop recorder}

A

39 year old woman was admitted for evaluation of recurrent syncope. A detailed workup, including invasive electrophysiological testing, was unremarkable. The patient initially refused implantation of a loop recorder, but finally agreed eight months later, after a further syncopal episode. Two years later, the patient was referred to our clinic for device explantation because of battery depletion. Without contacting our clinic, the patient had documented two short episodes of light headedness a few months after device implantation, corresponding with two episodes of non-sustained, fast, polymorphic ventricular tachycardia. The subsequently implanted automatic defibrillator revealed recurrent aymsptomatic episodes of nonsustained polymorphic ventricular tachycardia during the follow up of 10 months. The upper panel shows a normal sinus rhythm followed by a short run of non-sustained ventricular tachycardia (start and finish indicated by arrow) initiated by a couplet of ventricular extrasystoles (asterisks). The lower panel shows a continuation of the ECG; some sinus beats are followed by ventricular extrasystoles (bigemini) (paper speed $25 \mathrm{~mm} / \mathrm{s} ; 25 \mathrm{~mm} / \mathrm{mV}$ ).

In conclusion, potentially life threatening polymorphic ventricular tachycardia in this young woman without structural heart disease and recurrent syncope could only be diagnosed by means of an implantable loop recorder.

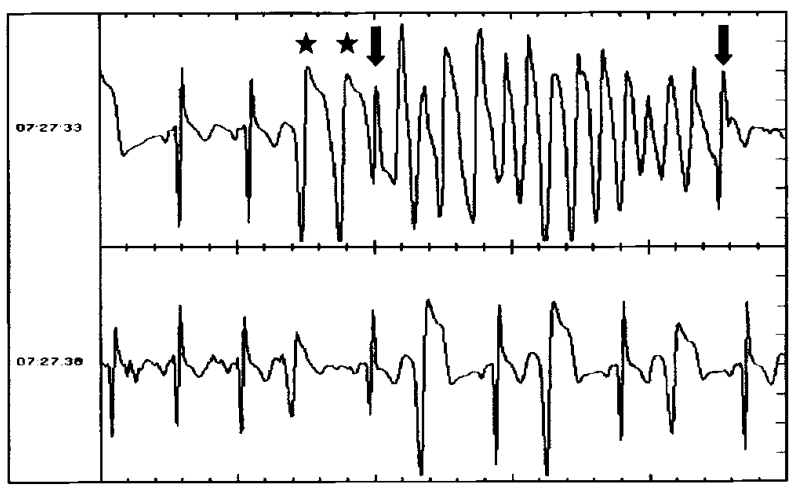

T Berger

F X Roithinger

F Hintringer

florian.hintringer@uklibk.ac.at 